\title{
Return of the Physician-Surgeon: The Role of Cardiovascular Surgeons in the Era of Transcatheter Valve Replacement/Repair Systems
}

\author{
Ha Lee, M.D., Suk-Won Song, M.D., Ph.D. \\ Department of Cardiovascular Surgery, Gangnam Severance Hospital, Yonsei University College of Medicine, Seoul, Korea
}

ARTICLE INFO

Received March 11, 2021, Accepted March 17, 2021

Corresponding author

Suk-Won Song

Tel 82-2-2019-3384, Fax 82-2-3461-8282, E-mail sevraphd@yuhs.ac, ORCID https://orcid.org/0000-0002-9850-9707

The national health burden of heart failure is increasing. The prevalence of heart failure in South Korea is approximately $1.5 \%$ to $2.0 \%$ (700 thousand to 1 million), and cardiologists estimate that this rate will double by 2040. A tremendous proportion of patients with valvular heart disease, which is the cause of heart failure, require valve repair or valve replacement. However, elderly patients are not able to undergo open heart surgery due to the high morbidity and mortality rate. This health issue is not limited to South Korea; instead, it is a global health problem that imposes a major public health burden. Therefore, 2 factors-the increasing prevalence of valvular heart disease and the increasing median population age-are driving global medical device companies to develop transcatheter valve replacement or repair systems.

The idea of an interventional approach for valve replacement or repair evolved starting in the late 1990s, and the first transcatheter aortic valve implantation (TAVI) was done in France in 2002. The next year, in 2003, the first transcatheter mitral valve repair (TMVr) system (MitraClip; Abbott, Santa Clara, CA, USA) was used for a procedure. In South Korea, the first TAVI case was done in 2010 and the first MitraClip case was performed in 2020. Despite numerous academic debates about the efficacy of TAVR and MitraClip [1], the popularity of the interventional approach is increasing. The Tendyne transcatheter mitral valve replacement (TMVR) system (Abbott) received a CE (Conformité Européenne) mark in 2020 and the
SUMMIT (Clinical Trial to Evaluate the Safety and Effectiveness of Using the Tendyne Mitral Valve System for the Treatment of Symptomatic Mitral Regurgitation, NCT03433274) trial is ongoing. Other TMVR systems such as Intrepid (Medtronic, Minneapolis, MN, USA) and EVOQUE (Edwards Lifesciences, Irvine, CA, USA) are being tested [2], and the transcatheter approach to the tricuspid valve is being researched [3].

With the advent of transcatheter valve replacement or repair, some cardiovascular surgeons have expressed forebodings about their future, as there is speculation that the future of cardiovascular surgery is threatened. But is this true? Are there any new opportunities for cardiovascular surgeons in this "bleak" era? With a couple critical points of view, we arrive at the conclusion that cardiovascular surgeons will instead have new opportunities.

Firstly, interventional procedures are becoming complicated. Cardiac interventions are not as simple as coronary interventions. The MitraClip involves a transseptal approach and Tendyne involves a transapical approach. In the strict sense, only cardiovascular surgeons can perform the transapical technique safely. The following questions must be answered regarding cardiac interventionist/cardiologists: (1) Can a cardiac interventionist, who can only perform the transseptal technique, offer optimal treatment to patients? (2) Can cardiologists be delicate while performing complicated operative techniques? Cardiologists are excellent at interpreting electrocardiographs, echocar- 
diographs, and angiographs, but they never see the real heart. They are unfamiliar with how the real heart looks and feels-instead, their expertise lies in interpreting the shadow of the heart. Without a knowledge of the physical reality of the heart, is it possible for them to perform complicated interventions?

Secondly, who will be the leader of the heart team? Everybody advocates for a multidisciplinary approach, according to which a single procedure requires many specialists. For example, during the MitraClip procedure, cardiac surgeons, cardiac interventionists, heart failure specialists and cardiac anesthetists are involved. However, there have not been any discussions about the leader. What if there is an emergency situation during a MitraClip procedure performed by a cardiac interventionist and a cardiac surgeon gets involved to overcome the situation by making independent decisions? Who has the responsibility in the field and who will explain the situation to the patient? Cardiologists rarely discuss the leader of the heart team. Here, then, we must wonder: are cardiologists trying to hide behind the concept of a "team approach" medicine?

In the past, there was no boundary between physicians and surgeons. The division between physicians and surgeons was just a byproduct of the development of modern medicine; before the advent of modern medicine, a physician who had meticulous hands became a surgeon and he or she was referred to as a physician-surgeon. We must recall the concept of a physician-surgeon in the current environment. Reductionism, which has been the methodology of modern medicine, has reached a critical point. Although reductionism gave rise to various divisions of medicine, with codes of professionalism in each department, we are now losing the sense of a holistic view of a patient. Imagine a physician-surgeon who can both perform open heart valve surgery and operate transcatheter valve replacement/ repair systems. He or she would be able to provide optimal treatment to a patient, unaffected by prejudices regarding the treatment modality. That is, the physician-surgeon-a cardiac surgeon who can also perform interventional approaches - can fulfill the holistic paradigm of post-modern medicine.

As treatments become more complicated and diverse, more leadership is required to make clear decisions in the clinical field. In our opinion, only cardiac surgeons are candidates for the heart team leader who can make the final decisions. Carl Schmitt, a German political scientist, stated in his book "Political theology" that the "sovereign's decisions are based upon the state of exception (emergency) [4]." Only cardiac surgeons are able to make decisions in emergency situations. Heart failure specialists and cardiac interventionists are only familiar with rules and indications. When a clinical situation changes, they seek out cardiac surgeons, because they know that we are trained as professionals to handle the state of exception and can make decisions to overcome the situation. Cardiac surgeons must know how the MitraClip works in order to lead the heart team and to provide optimal care for the patient. This does not mean that cardiac surgeons should just read articles about the MitraClip; instead, cardiac surgeons must gain hands-on experience with the MitraClip. Here is where we, as cardiac physician-surgeons, should start.

\section{Conflict of interest}

No potential conflict of interest relevant to this article was reported.

\section{ORCID}

Ha Lee: https://orcid.org/0000-0003-3976-8554

Suk-Won Song: https://orcid.org/0000-0002-9850-9707

\section{References}

1. Lindenfeld J, Abraham WT, Grayburn PA, et al. Association of effective regurgitation orifice area to left ventricular end-diastolic volume ratio with transcatheter mitral valve repair outcomes: a secondary analysis of the COAPT trial. JAMA Cardiol 2021 Feb 3 [Epub]. https://doi.org/10.1001/jamacardio.2020.7200.

2. Hensey M, Brown RA, Lal S, et al. Transcatheter mitral valve replacement: an update on current techniques, technologies, and future directions. JACC Cardiovasc Interv 2021;14:489-500.

3. Fam NP, von Bardeleben RS, Hensey M, et al. Transfemoral transcatheter tricuspid valve replacement with the EVOQUE system: a multicenter, observational, first-in-human experience. JACC Cardiovasc Interv 2021;14:501-11.

4. Schmitt C. Political theology: four chapters on the concept of sovereignty. Chicago (IL): University of Chicago Press; 2005. 